\title{
Normal Esophagus Misdiagnosed as a Thyroid Nodule by Non- Radiologist Reading Thyroid US: Case Report
}

\author{
Karla E Abundiz-Bibiano1*, Roberto Rivera-Villanueva ${ }^{2}$, Ernesto \\ Roldan-Valadez ${ }^{3}$, Rogerio Santiago-Herrera ${ }^{2}$ \\ ${ }^{1}$ Radiology Resident at the Posgraduate School of Naval Sanity, Universidad Naval \\ and the Hospital General Naval de Alta Especialidad, Secretaria de Marina Armada de \\ México (SEMAR).
}

${ }^{2}$ Specialist in Radiology, Naval General Hospital of High Specialty (HOSGENAES), Mexico

${ }^{3}$ Directorate of Research, General Hospital of Mexico, Mexico

*Corresponding author: Karla E Abundiz-Bibiano, Hospital General Naval de Alta Especialidad, Secretaria de Marina Armada de México (SEMAR). Calz de la Virgen, Presidentes Ejidales 1ra Secc, 04470 Ciudad de Mexico, CDMX, Mexico, Tel: +52-155-4478-2544; Email: abundizkarla@hotmail.com

\section{Abstract}

Ultrasound (US) of the thyroid gland represents one of the first diagnostic approaches in the characterization of thyroid nodules. Specific US features allow the radiologist to differentiate malignant versus benign pathology. We report a case of a patient who was sent for a biopsy to the radiology department for the diagnosis of a left thyroid nodule with microcalcifications and irregular borders. After a US evaluation, the attending radiologist reported non-existing lesions in the thyroid gland, some previous images from the esophagus were what a non-radiologist physician observed at an earlier thyroid ultrasound and considered a thyroid nodule with an indication of biopsy. If the low-moderate level of agreement is found between the radiologists interpreting US findings of neck structures, then non-radiologist interpreting US images for their patients may need further education. Clinicians should be aware of the characteristics of normal anatomic structures that can simulate thyroid nodules when they explain ultrasonography to their patients.

Keywords: Esophagus; Ultrasonography; Thyroiditis; Nodules; Microcalcifications; Biopsy; Hyperaldosteronism

Abbreviations: ACR: American College of Radiology; TI-RADS: Thyroid Imaging Reporting and Data System.

\section{Introduction}

Ultrasound (US) of the thyroid gland represents one of the first diagnostic approaches in the characterization of thyroid nodules. Specific US features allow the radiologist to differentiate malignant versus benign pathology; also US findings support the decision making of the specialist 


\section{Clinical Radiology \& Imaging Journal}

regarding the follow-up of a detected lesion and the need for biopsy. Currently, the American College of Radiology (ACR) Thyroid Imaging Reporting and Data System (TIRADS) is used as a risk stratification system of thyroid nodules based on the assignment of ultrasound characteristics [1]. This system proposes standardized criteria among radiologists and clinicians, not forgetting the variability that may exist according to the level of experience.

We report a case of a patient who was sent for a biopsy to the radiology department for the diagnosis of a left thyroid nodule with microcalcifications and irregular borders. After a US evaluation, the attending radiologistreported non-existing lesions in the thyroid gland, some previous images from the esophagus were what anon-radiologist physician observed at an earlier thyroid ultrasound and considered a thyroid nodule with an indication of biopsy.

\section{Case Report}

A 46-year-old woman was sent to radiology and imaging department to perform thyroid US to guide a fine needle biopsy due to a previous diagnosis of hypothyroidism. The patientunderwent former Neck US two months before that reported no abnormalities. However, the images of the US were considered with a left thyroid nodule with irregular margins and microcalcifications by the treating physician (nonradiologist).

A 10-year experienced radiologist performed the second neck US examination used a Samsung Medison model RS80A with Linear Transducer of 5-10 MHz. The US showed the thyroid gland with a thickness of $2.3 \mathrm{~mm}$ at the isthmus level. Measures of the right lobe were $35 \mathrm{x}$ $11 \mathrm{x} 14 \mathrm{~mm}$ (longitudinal axis, transverse and anteroposterior respectively), and left lobe $36 \times 11 \times 10$ $\mathrm{mm}$ (longitudinal axis, transverse and anteroposterior respectively. The thyroid parenchyma was observed typically homogeneous granular with the usual anatomical relationships, such as infrahyoid muscles, common carotid, jugular and esophagus (Figure 1).

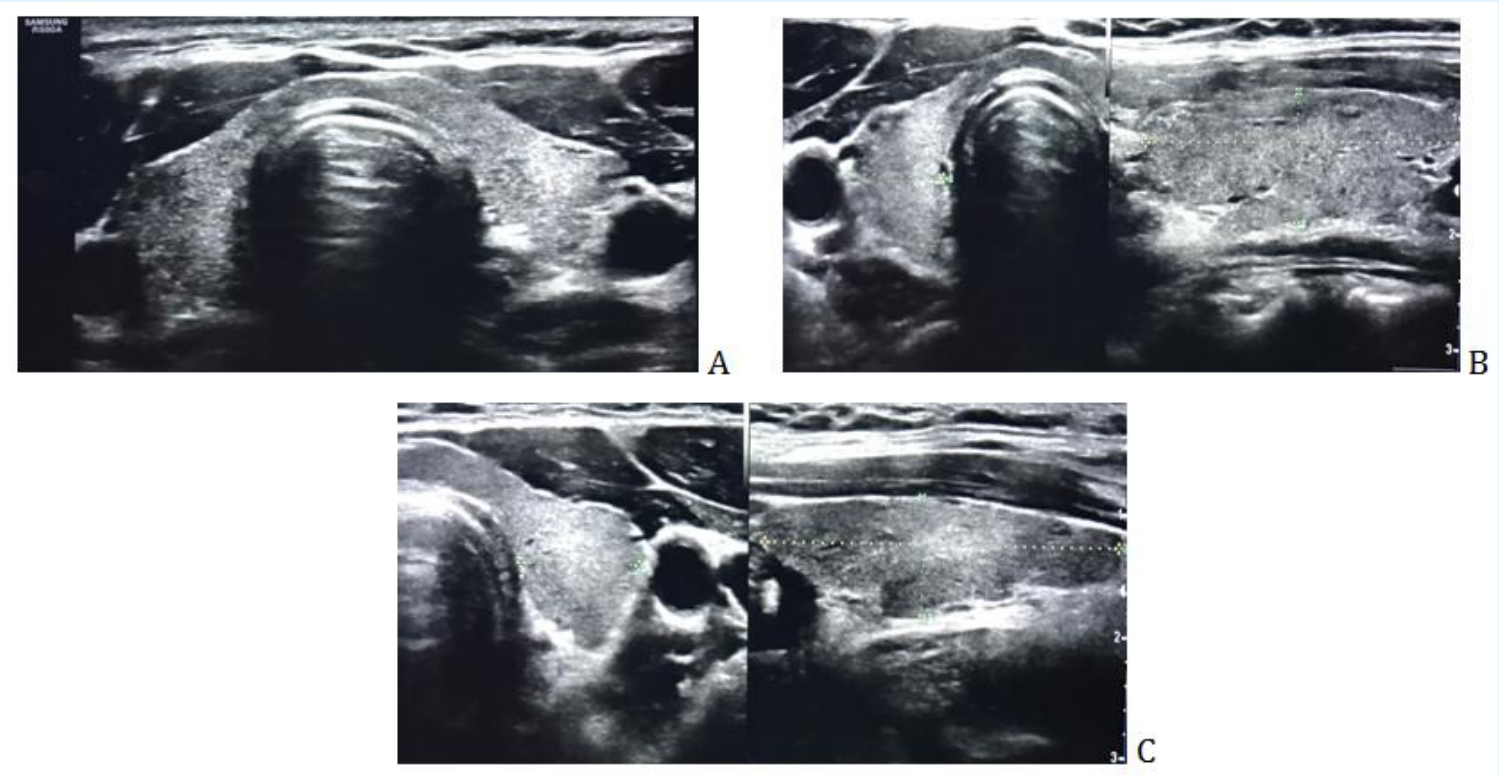

Figure 1: The thyroid gland in longitudinal and transverse planes. A) The whole thyroid gland in cross section. B) Right thyroid lobe. C) Left thyroid lobe. C) Oblique view of the left thyroid lobe.

Because laboratory exams showed normal parameters, another US neck evaluation was performed to confirm the non-existence of the thyroid nodule observed by the treating physician. After commenting on the imaging findings with the endocrinology department, the indication of thyroid biopsy was postponed concluding that an image from the esophagus had been misdiagnosed as a thyroid nodule. During the physical examination, the patient referred difficulty in swallowing,but no masses were palpated at neck level; 


\section{Clinical Radiology \& Imaging Journal}

she was able to observe the characteristics of her esophagus in the US and her swallowing dynamically, she received an explanation of the null existence of a nodule (Figure 2). The patient was reassessed by attending physician in the outpatient clinic. Currently, she has the diagnosis of probable primary hyperaldosteronism, gastritis, and esophageal motility disorder. The patient will continue her appointments at the Endocrinology department with scheduled tests for plasma aldosterone concentration and plasma renin activity; her thyroid hormone levels are normal; she will be evaluatedin the gastroenterology department.
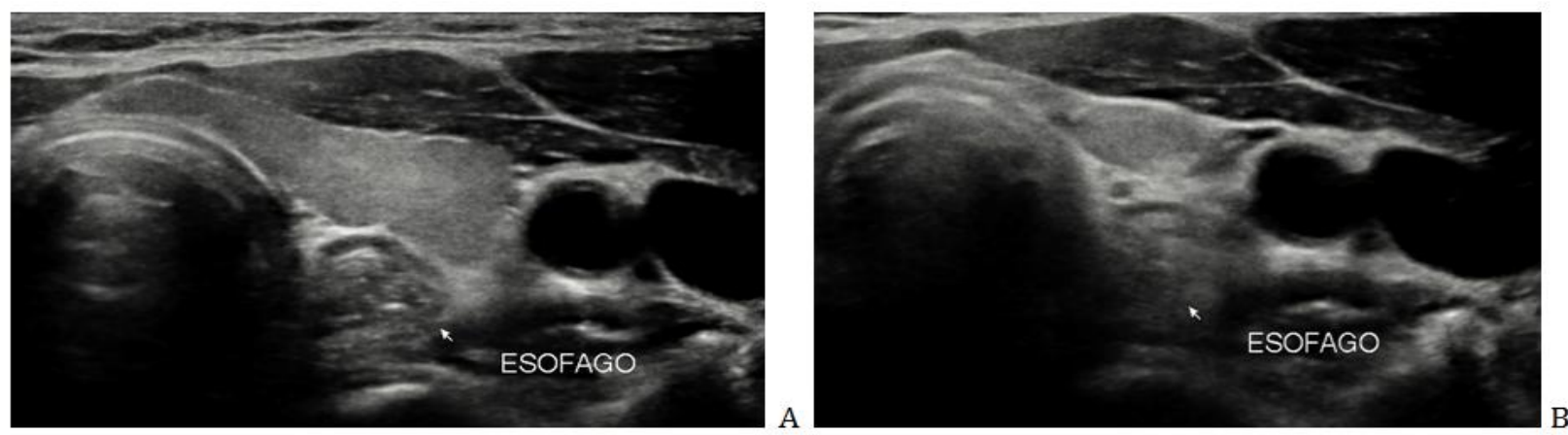

Figure 2: A) Left thyroid lobe with visualization of the esophagus, which gives the impression of calcium images inside. B) Swallowing patient, no longer observing esophagus as a previous image.

\section{Discussion}

Interpretations of US features of the thyroid change the level of suspicion of a nodule from benign to malignancy. However, there are several reports of nonradiologists (one of the dominant specialties is otolaryngologists) that after a routine examination of neck ultrasound and enhanced CT, there is a misdiagnosis of a neck mass. Pyriform sinus fistula has been misdiagnosed as suppurative lymphadenitis, thyroglossal duct cyst (and performed excision of thyroglossal duct cyst), as second branchial cleft fistula, suppurative thyroiditis, and even thyroid neoplasms (two cases underwent partial thyroidectomy) [2].

Other reports of esophageal-related diseases detected by ultrasound examination and misdiagnosed as thyroid diseases preoperatively include cervical esopha geal cancer metastasized to thyroids that initially was misdiagnosed as thyroid cancer, and Zenker's diverticulum initially diagnosed as nodular goiter; all patients reported in that study did not have any clinical feature of esophageal diseases [3].

It is worth mentioning that the agreement in interpretation "among radiologists" has been reported with significant variations. For example, the margin and other types of echogenic foci reported in a previous study, showed kappa values ranging from 0.25 to 0.39 , shape $\kappa=$ 0.61 , macrocalcifications $\kappa=0.73$, and the inter observer agreement regarding biopsy recommendation with a low $\kappa=0.22[1]$.

In conclusion, if the low-moderate level of agreement is found between the radiologists interpreting US findings of neck structures, then non-radiologist interpreting US images for their patients may need further education. The adoption of ACR TI-RADS improves agreement for recommending a biopsy. To avoid misdiagnosis of malignancy, clinicians should be aware of the characteristics of normal anatomic structures that can simulate thyroid nodules when they interpret ultrasonography to their patients. Likewise, surgery of the thyroid studies should be performed in expert hands to avoid confusion that can be prevented with the use of ACRTI-RADS in a standardized manner, thereby increasing confidence and good patient-physician relationship, as well as the unification of criteria between the different specialties in an interdisciplinary way, avoiding causing doubts in the patient about her illness.

\section{References}




\section{Clinical Radiology \& Imaging Journal}

1. Hoang JK, Middleton WD, Farjat AE, Teefey SA, Abinanti N, et al. (2018) Interobserver Variability of Sonographic Features Used in the American College of Radiology Thyroid Imaging Reporting and Data System. AJR Am J Roentgenol 211(1): 162-167.

2. Ma J, Ming C, Lou F, Wang ML, Lin K, et al. (2018) [Misdiagnosic analysis and treatment of pyriform sinus fistula in children]. Zhonghua Er Bi Yan Hou Tou Jing Wai Ke Za Zhi 53(5): 381-384.

3. Ye-huan L, Shi-xu L, Yi-li Z, Ou-chen W, Xiao-hua Z (2015) Unexpected esophageal diseases appeared in thyroid resections. World J Surg Oncol 13: 131.

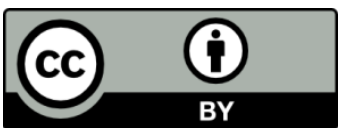

